Revue internationale P.M.E.

Économie et gestion de la petite et moyenne entreprise

\title{
Vers une typologie multicritère des PME
}

\section{Pierre-André Julien}

Volume 3, numéro 3-4, 1990

URI : https://id.erudit.org/iderudit/1007988ar

DOI : https://doi.org/10.7202/1007988ar

Aller au sommaire du numéro

Éditeur(s)

Presses de l’Université du Québec

ISSN

0776-5436 (imprimé)

1918-9699 (numérique)

Découvrir la revue

Citer cette note

Julien, P.-A. (1990). Vers une typologie multicritère des PME. Revue

internationale P.M.E., 3(3-4), 411-425. https://doi.org/10.7202/1007988ar d'utilisation que vous pouvez consulter en ligne.

https://apropos.erudit.org/fr/usagers/politique-dutilisation/ 


\title{
Notes de lecture Vers une typologie multicritère des PME
}

\author{
Pierre-André JULIEN \\ GREPME \\ Université du Québec à Trois-Rivières
}

\section{Introduction}

Comme le rappelait André Cibert dans les «Perspectives» du numéro précédent de la Revue, la plupart des spécialistes considèrent qu'une des plus grandes difficultés que l'on rencontre en étudiant les PME est l'extrême hétérogénéité qui existe entre elles : quoi de commun, en effet, entre le mineur licencié qui ouvre une épicerie dans son village ou l'étudiant qui, en terminant ses études, loue quelques machines à photocopier pour offrir un tel service près de son collège et l'ouvrier spécialisé qui, fort de son expérience dans une grande entreprise, décide de partir à son propre compte ou encore l'ingénieur qui monte dans un incubateur une entreprise électronique très spécialisée ? Au départ, on différencie les PME du secteur manufacturier de celles du commerce et des services, sachant qu'il y a peu de ressemblances entre elles. Mais on se rend compte aussi que les petites entreprises franchisées ou liées à une bannière ont des particularités différentes des autres plus indépendantes. De même, on considère que les artisans, avec 2 ou 3 employés, fonctionnent avec une toute autre logique que les entreprises ayant 100 ou 150 employés. Enfin, on commence de plus en plus à distinguer les moyennes entreprises (au fonctionnement souvent proche des grandes), des petites et, évidemment, des très petites firmes.

Cette hétérogénéité (ainsi qu'une plus grande volatilité causée par une création continue et de nombreuses disparitions dans les premières années d'exis- 
tence) expliquerait en partie le retard des chercheurs à se pencher sur les PME et la difficulté d'en tirer des théories et des concepts adéquats différents de ceux appliqués à la grande entreprise. Si la loi des grands nombres peuts'appliquer aux comportements des grandes entreprises, dans lesquelles on retrouve plusieurscentaines de gestionnaires et de cadres aux profils variés, et donc avec une probabilité élevée de retrouver les mêmes «moyennes», cela s'avère presque impossible dans des petites entreprises avec un ou deux cadres au profil psychologique souvent très différent d'une entreprise à l'autre ou fonctionnant dans des organisations fort disparates.

Pourtant, la science fonctionne avant tout par généralisation et par regroupement, et le domaine des PME ne peut y échapper, même si cela semble particulièrement difficile à faire. C'est pourquoi, comme on le sait, un grand nombre de chercheurs ont tenté de construire des typologies opérationnelles des PME et des petites entreprises pour justement retrouver ces ressemblances ou comportements «moyens».

Évidemment, on retrouve parmi celles-ci les typologies traditionnelles basées sur des critères quantitatifs. Mais devant leurs limites, plusieurs auteurs ont voulu aller plus loin en tenant compte du dirigeant de l'entreprise, de ses comportements managériaux ou organisationnels, ou encore de l'évolution de la firme ou de ses relations avec les différents marchés.

\section{Les typologies quantitatives}

Pour toutes les typologies, il faut d'abord bien distinguer les entreprises des établissements. Lorsqu'on parle d'entreprises ou de PME, on se réfere à des centres de contrôle, avec une propriété délimitée. Pour les très petiteset les petites entreprises, cela présente peu de problèmes : l'établissement se confond le plus souvent avec l'entreprise, et la propriété est souvent bien connue. Mais cela est moins évident dans le cas de la moyenne entreprise. Aussi, un des premiers critères porte sur la notion juridique de l'indépendance de l'entreprise; un petit établissement contrôlé par une grande entreprise n'est donc pas une PME.

Mais cette notion «d'indépendance», ne résout pas tous les problèmes. Qu'en est-il, par exemple, des franchises ou des bannières ? Certaines entreprises franchisées reçoivent peu de services et peuvent être considérées au même titre que les «bannières» regroupant des entreprises indépendantes qui se sont associées pour augmenter leur pouvoir d'achat et de mise en marché; d'autres firmes relèvent d'une simple décision financière du propriétaire, alors que toute la gestion et la stratégie sont orientées par l'entreprise-franchiseur : on peut les considérer dans ce cas comme un type de filiales. Il en est de même des sous-traitants «de capacité» liés à un seul donneur d'ordre: leur indépendance pose problème ${ }^{1}$; alors que les sous-traitants «de

1 Jacques Houssiaux (1957) parle ici de «quasi-intégration». 
spécialité» ont un certain pouvoir sur le ou les donneur(s) d'ordre. Aussi chaque cas doit être étudié à son mérite.

Nonobstant cette notion d'indépendance, on peut dire que les typologies des PME les plus connues et les plus souvent utilisées réfèrent à des données quantitatives d'emplois, d'actifs ou de chiffre d'affaires. Par exemple, le ministère québécois de l'Industrie, du Commerce et de la Technologie, divise les PME ainsi :

Tab. 1 Classification des entreprises manufacturières

\begin{tabular}{|lll|}
\hline Nombre d'employés & $\begin{array}{l}\text { Montant des actifs en } \$ \\
\text { (en millions de FF) }\end{array}$ & Type d'entreprise \\
\hline $0-49$ & Inférieurs à $3000000(13,5)$ & Petite \\
$50-200$ & 3000000 à moins de & Moyenne \\
& 12000000 (13,5 à 55) & \\
$200-499$ & 12000000 à moins de & Grande \\
& 30000000 (55 à 135) & Très grande \\
\hline
\end{tabular}

Source : Les PME au Québec : état de la situation, Québec, 1988.

Tab. 2 Classification des entreprises autres que manufacturières

\begin{tabular}{|lll|}
\hline Nombre d'employés & $\begin{array}{l}\text { Ventes annuelles en } \$ \\
\text { (en million de FF) }\end{array}$ & Type d'entreprise \\
\hline $0-49$ & Moins de $2000000(9)$ & Petite \\
$50-99$ & 2000000 à moins de & Moyenne \\
100 et plus & $20000000(9$ à 90$)$ & Grande \\
\hline
\end{tabular}

Source : Ibidem

Mais la taille (nombre d'employés) diverge selon les pays, et même à l'intérieur de ceux-ci, selon les programmes gouvernementaux. Ainsi, M. Glader (1981) donne ces chiffres pour l'emploi dans les PME de divers pays : 
Tab. 3 Classification des firmes, selon la taille, de quelques pays industrialisés

\begin{tabular}{|lccc|}
\hline Pays & $\begin{array}{c}\text { Petites } \\
\text { firmes }\end{array}$ & $\begin{array}{c}\text { Nombre d'employés } \\
\text { Moyennes } \\
\text { firmes }\end{array}$ & $\begin{array}{c}\text { Grandes } \\
\text { firmes }\end{array}$ \\
Autriche & $1-9$ & $10-100$ & $>101$ \\
Belgique & $1-50$ & $51-200$ & $>201$ \\
Danemark & $1-50$ & $51-200$ & $>201$ \\
Etats-Unis & $1-250$ & $250-500$ & $>501$ \\
Finlande & $1-50$ & $51-200$ & $>201$ \\
France & $1-49$ & $50-499$ & $>500$ \\
Grande-Bretagne & $1-50$ & $51-200$ & $>201$ \\
Japon & $1-49$ & $50-500$ & $>501$ \\
Norvège & $1-20$ & $21-100$ & $>101$ \\
République féd. allem. & $1-49$ & $50-499$ & $>500$ \\
Suisse & $1-20$ & $21-100$ & $>101$ \\
\hline
\end{tabular}

Source : M. Glader, «Research on small enterprises", Cahier de recherche, Université de Umeá, 1981.

On retrouve d'autres données dans D.J. Storey (1983) pour certains pays en voie de développement, comme on peut le voir au tableau 4 pour les pays asiatiques, avec diverses spécifications selon le type de programme gouvernemental :

Tab. 4 Classification des petites firmes dans quelques pays asiatiques

\begin{tabular}{|llll|}
\hline Pays & \multicolumn{2}{c|}{ Nombre d'employés } & Actifs fixes \\
Indonésie & 1) & Moins de 19 & \\
& 2) & Moins de 10 & Moins de Rp 500000 \\
Malaisie & 1) & Moins de 25 & Moins de M $\$ 250000$ \\
& 2) & Moins de 50 & \\
Philippines & 1) & 5 à 99 & R100 000 à $R 1$ million \\
Singapour & 2) & & Moins de $R 1$ million \\
Thaïlande & 1) & Moins de 50 & - \\
& 2) & Moins de 100 & Moins de $\$ \$ 2$ millions \\
& 1) & moins de 50 & \\
& 2) & 10 à 49 & \\
& 3) & & Moins de 2 millions baht \\
\hline
\end{tabular}

Source : D.J. Storey (éd.), The Small Firm : an International Survey, New York, St. Martin Press, 1983.

Ces spécifications quantitatives sont monnaie courante. Plusieurs pays ont toutefois différentes normes selon les programmes. Ainsi la «Small Business 
Administration» américaine accepte pour certains de ses programmes de subventionner des entreprises ayant jusqu'à 1000 employés, alors que pour d'autres, ce nombre d'employés est moins de 500.

De toute façon, ces typologies quantitatives, d'unepart, relèvent de l'approche économique traditionnelle qui se refuse à pénétrer à l'intérieur de la boîte noire de l'entreprise et ne touche qu'aux éléments les plus apparents; elles sont toutefois les premières disponibles et peuvent servir, comme nous l'avons dit, à répondre aux besoins de critères pour l'application des programmes d'aide gouvernementaux. D'autre part, pour les chercheurs, elles sont souvent une première porte d'entrée pour obtenir des échantilllons qui seront étudiés plus attentivement par la suite.

Évidemment, cette mesure statistique comporte différents problèmes, à savoir, entre autres, ce que l'on entend par «nombre d'employés» : employés permanents, à temps partiel, saisonniers, semi-cadres, etc.; on réussit toutefois à résoudre pas trop mal ces problèmes par quelques définitions et calculs. Mais cela ne résout pas le cas des différences sectorielles : par exemple, on peut considérer qu'un atelier d'usinage de 50 employés est, dans son secteur, une entreprise moyenne; alors qu'une PME de 100 employés dans le secteur du vêtement est de petite taille.

Les différences sont souvent grandes entre les industries à travail intensif et celles à capital intensif. C'est pourquoi on ajoute au nombre d'employés, comme c'est le cas au tableau 1, la mesure des actifs. Mais qu'en est-il de la taille des firmes aux actifs passablement dépréciés à côté de firmes aux actifs neufs? En tenant compte du chiffre d'affaires, de la valeur ajoutée ou des ventes (comme on le voit, dans ce dernier cas, au tableau 2), on peut surmonter cet obstacle. Mais encore ici, le chiffre d'affaires peut varier considérablement selon les branches industrielles en croissance ou à large marché par rapport à celles à marché étroit; de plus, il est souvent camouflé pour des raisons fiscales; les ventes sont fonction de la conjoncture ou peuvent varier considérablement selon les saisons; la valeur ajoutée peut être difficile à évaluer; etc. D'ailleurs, même s'il y a une certaine corrélation entre ces différentes mesures de taille, elles ne sont pas complètement interchangeables (Nguyen et Belhumeur, 1895).

Le «Bolton Committee» et le gouvernement américain ont ainsi ajouté la notion de part de marché : une PME ne doit pas contrôler une part importante du marché. Mais dans ce cas, parle-t-on de marché local, régional, ou national ?

Ainsi, les typologies quantitatives, pourtant si faciles d'approche, demeurent fort criticables et peuvent être utilisées uniquement comme première approximation ${ }^{2}$. On doit donc ajouter d'autres critères pour distinguer les différents types de PME.

2 D'autres problèmes peuvent survenir de la qualité des données statistiques, en particulier lorsqu'elles tiennent compte des toutes petites entreprises ou du moins de celles ayant moins de 20 employés. En fait, il est rare que deux sources différentes arrivent aux mêmes résultats. Voir à ce propos Brown et Phillips (1989). 


\section{Les typologies multicritères ou qualitatives}

Ainsi, plusieurs chercheurs ont tenté de dépasser ces typologies primaires. En pénétrant à l'intérieur de la boîte noire et en tenant compte de la relation de l'entreprise avec son environnement, ils ont ainsi adopté une approche beaucoup plus managériale et organisationnelle.

On peut diviser ces typologies plus complexes en quatre grands groupes, soit celles qui s'appuient sur le type d'origine ou de propriété de l'entreprise, celles qui introduisent les stratégies ou les objectifs de la direction, celles qui se basent sur l'évolution ou le stade de développement ou d'organisation de la firme et, enfin, celles qui touchent au secteur ou au type de marché dans lequel la firme évolue. Les figures suivantes présentent, avec quelques éléments connexes, ces différentes typologies selon ce regroupement, certaines relevant d'analyses théoriques alors que d'autres ont été «testées» auprès d'échantillons de PME (notées T).

Le premier groupe de typologies (Deeks, 1973; Gélinier et Gaultier, 1974; et Barry, 1978) est basé sur un critère très simple. Ce dernier est aussi utilisé dans un grand nombre de typologies associées aux différents types d'entrepreneurs et dont nous ne parlons pas ici (Smith et Miner, 1983; Lorrain et Dussault, 1988). On peut penser que l'origine ou le type de propriété affecte, jusqu'à un certain point, la forme d'organisation de l'entreprise, ou du moins son évolution à long terme.

\subsection{Typologies selon le type de propriété}

\begin{tabular}{|c|c|c|c|c|c|}
\hline $\begin{array}{l}\text { J. Deeks (1973): } \\
\text { artisan }\end{array}$ & $\begin{array}{l}\text { Propriétaire- } \\
\text { dirigeant }\end{array}$ & $\begin{array}{l}\text { Propriétaire- } \\
\text { participant }\end{array}$ & Actionnaire & $\begin{array}{l}\text { Propriétaire- } \\
\text { dirigeant } \\
\text { fondateur }\end{array}$ & $\begin{array}{l}\text { Propriétaire- } \\
\text { dirigeant } \\
\text { héritier }\end{array}$ \\
\hline $\begin{array}{l}\text { O. Gélinier ot } \\
\text { A. Gaultier (1974) } \\
\text { ou B. Barry (1978): }\end{array}$ & $\begin{array}{l}\text { Familiale } \\
\text { (transmise à } \\
\text { un membre de } \\
\text { la famille) }\end{array}$ & & & \multicolumn{2}{|c|}{$\begin{array}{l}\text { Personnelle } \\
\text { (les fondateurs } \\
\text { détiennent toujours } \\
\text { le contrôle) }\end{array}$} \\
\hline
\end{tabular}

Le deuxième groupe fait le pont entre les intérêts des propriétaires-dirigeants ou leur stratégie et l'organisation ou le potentiel des firmes. Les plus anciennes typologies de ce groupe (Liles, 1974; Stanworth et Curran, 1976; Filey et Aldag, 1978) sont aussi issues des typologies d'entrepreneurs tout en faisant le lien avec leur évolution en termes de taille (de toutes petites entreprises ou artisanales à des entreprises un peu plus grandes et mieux structurées ou ayant une organisation plus professionnelle). Les plus récentes (Cooper et Dunkelberg, 1982; Miller et Friesen, 1982; Carland et alii, 1984) ajoutent à ces éléments la stratégie suivie par la direction. Enfin, M. Marchesnay (1988) relie cette stratégie aux intérêts de l'entrepreneur pour l'indépendance et l'impact sur le marché du point de vue de la croissance ou de la stabilité. 


\subsection{Typologies selon le type d'objectifs de la direction, la straté- gie poursuivie ou le potentiel de la firme}

\begin{tabular}{|c|c|c|c|c|}
\hline P. Liles (1974): & $\begin{array}{l}\text { Marginale } \\
\text { (traditionnelle) }\end{array}$ & \multicolumn{2}{|c|}{$\begin{array}{l}\text { Intéressante } \\
\text { (taille et croissance } \\
\text { à venir) }\end{array}$} & $\begin{array}{l}\text { A haut potentiel (à } \\
\text { croissance rapide) }\end{array}$ \\
\hline $\begin{array}{l}\text { M. Stanworth et : } \\
\text { J. Curran (1976): }\end{array}$ & $\begin{array}{l}\text { Artisanale } \\
\text { (satisfaction } \\
\text { intrinsèque) }\end{array}$ & \multicolumn{2}{|c|}{$\begin{array}{l}\text { Entrepreneuriale } \\
\text { classique (profit) }\end{array}$} & $\begin{array}{l}\text { Managériale } \\
\text { (reconnaissance } \\
\text { par la performance) }\end{array}$ \\
\hline $\begin{array}{l}\text { A. Filey of } \\
\text { R. Aldag (1978): }\end{array}$ & $\begin{array}{l}\text { Artisanale } \\
\text { (conventionnelle, } \\
\text { risques faibles, } \\
\text { stratégie de survie) }\end{array}$ & \multicolumn{2}{|c|}{$\begin{array}{l}\text { Promotionnelle } \\
\text { (entreprenariale, } \\
\text { technicienne, } \\
\text { innovatrice, } \\
\text { s. de croissance) }\end{array}$} & $\begin{array}{l}\text { Administrative } \\
\text { (professionnelle, } \\
\text { structurée, } \\
\text { risques +élevés, } \\
\text { planifiée) }\end{array}$ \\
\hline $\begin{array}{l}\text { A. Cooper et } \\
\text { C. Dunkelberg } \\
(1982, \mathrm{~T}) \text { : }\end{array}$ & $\begin{array}{l}\text { De type artisanal } \\
\text { (autonomie) }\end{array}$ & \multicolumn{2}{|c|}{$\begin{array}{l}\text { Recherche de } \\
\text { l'indépendance }\end{array}$} & $\begin{array}{l}\text { Orientée vers la } \\
\text { croissance }\end{array}$ \\
\hline $\begin{array}{l}\text { D. Miller et } \\
\text { P.H. Friesen } \\
\text { (1982): }\end{array}$ & \multicolumn{2}{|c|}{$\begin{array}{l}\text { Entreprenariale } \\
\text { (recherchant diverses } \\
\text { initiatives) }\end{array}$} & $\begin{array}{l}\text { Conservatri } \\
\text { (traditionne }\end{array}$ & \\
\hline $\begin{array}{l}\text { J. Carland et alii } \\
\text { (1984): }\end{array}$ & \multicolumn{2}{|c|}{$\begin{array}{l}\text { Entreprenariale } \\
\text { (profit, croissance, } \\
\text { créativité) }\end{array}$} & \multicolumn{2}{|c|}{$\begin{array}{l}\text { De gérance } \\
\text { (recherche de buts } \\
\text { personnels) }\end{array}$} \\
\hline $\begin{array}{l}\text { M. Marchesnay } \\
\text { (1988): }\end{array}$ & \multicolumn{2}{|c|}{$\begin{array}{l}\text { C.A.P. } \\
\text { (croissance, autonomie, } \\
\text { pérennité) }\end{array}$} & \multicolumn{2}{|c|}{$\begin{array}{l}\text { P.I.C. } \\
\text { (pérennité, indépendance, } \\
\text { croissance) }\end{array}$} \\
\hline
\end{tabular}

Le troisième groupe rassemble le plus grand nombre de typologies dont, à notre connaissance, les plus anciennes (1969). Un premier sous-groupe (Greiner, 1972; Churchill et Lewis, 1984; Vargas, 1984) est directement issu de l'idée du cycle de vie des entreprises, déjà bien discutée chez Edith Penrose, par analogie avec la biologie ${ }^{3}$ sinon la philosophie de l'histoire, et reprise par la suite par Mueler (1972). En cela, il est rattaché à la philosophie positiviste selon laquelle, dans ce cas-ci, toutes les entreprises suivent le même sentier d'évolution en naissant d'abord petites et en passant ensuite par différents stades jusqu'à devenir grandes sinon très grandes, à moins de périr en chemin. En ce sens, ces typologies posent plusieurs problèmes puisqu'au contraire, d'une part, les PME demeurent pour la plupart petites, même après 50 ou 100 ans, ou encore certaines entreprises naissent déjà grandes et bien organisées, et, d'autre part, il existe différents sentiers d'évolution pour les entreprises selon les secteurs, les marchés, les conjonctures, etc.

3 A partir d'un article paru en 1952, et repris par la suite dans son ouvrage bien connu paru en 1957 (Penrose, 1952; 1957).

4 En particulier chez les philosophes de l'histoire comme Spencer, Huxley ou Toynbee. 


\subsection{Typologies selon l'organisation et la crolssance ou le déve- loppement (la taille)}

\begin{tabular}{|c|c|c|c|c|c|c|c|c|}
\hline D. Thain (1969): & \multirow{2}{*}{\multicolumn{3}{|c|}{$\begin{array}{l}\text { Stade } 1 \\
\text { une unité } \\
\text { (un seul homme) } \\
\text { Stade } 1 \\
\text { (supervision directe) }\end{array}$}} & \multicolumn{3}{|c|}{$\begin{array}{l}\text { Stade } 2 \\
\text { une unité avec } \\
\text { spécialisation }\end{array}$} & \multicolumn{2}{|c|}{$\begin{array}{l}\text { Stade } 3 \\
\text { décentralisée } \\
\text { par fonction }\end{array}$} \\
\hline L. Steinneetz (1969): & & & & \multicolumn{3}{|c|}{$\begin{array}{l}\text { Stade } 2 \\
\text { (supervision } \\
\text { supervisée) }\end{array}$} & \multicolumn{2}{|c|}{$\begin{array}{l}\text { Stade } 3 \text { et plus } \\
\text { (direction complexe : } \\
\text { vers la GE) }\end{array}$} \\
\hline $\begin{array}{l}\text { L. Greiner } \\
\text { (1972) et } \\
\text { G. Vargas } \\
\text { (1984): }\end{array}$ & \multicolumn{2}{|c|}{$\begin{array}{l}\text { Stade } 1 \\
\text { (crise de } \\
\text { leadership: } \\
\text { créativité) }\end{array}$} & \multicolumn{2}{|c|}{$\begin{array}{l}\text { Stade } 2 \\
\text { (crise d'auto- } \\
\text { nomie: } \\
\Delta \text { direction) }\end{array}$} & \multicolumn{2}{|c|}{$\begin{array}{l}\text { Stade } 3 \\
\text { (crise de } \\
\text { contróle : } \\
\text { délégation) }\end{array}$} & $\begin{array}{l}\text { Stade } 4 \\
\text { (crise de } \\
\text { bureaucratie : } \\
\text { coordination) }\end{array}$ & $\begin{array}{l}\text { Stade } 5 \\
\text { (crise...: } \\
\Delta \text { par collabo- } \\
\text { ration) }\end{array}$ \\
\hline $\begin{array}{l}\text { C. Kroeger } \\
\text { (1974): }\end{array}$ & \multicolumn{2}{|c|}{$\begin{array}{l}\text { Initiation } \\
\text { (idéateur) }\end{array}$} & \multicolumn{2}{|c|}{$\begin{array}{l}\text { Développement } \\
\text { (organisateur) }\end{array}$} & \multicolumn{2}{|c|}{$\begin{array}{l}\text { Croissance } \\
\text { (implantateur) }\end{array}$} & $\begin{array}{l}\text { Maturité } \\
\text { (gestionnaire) }\end{array}$ & $\begin{array}{l}\text { Déclin (suc- } \\
\text { cesseur réor- } \\
\text { ganisateur). }\end{array}$ \\
\hline $\begin{array}{l}\text { F. Webster } \\
\text { (1976): }\end{array}$ & \multicolumn{2}{|c|}{$\begin{array}{l}\text { Pré- } \\
\text { lancement }\end{array}$} & \multicolumn{2}{|c|}{$\begin{array}{l}\text { Stade du } \\
\text { travail collec- } \\
\text { tif et de l'en- } \\
\text { thousiasme }\end{array}$} & \multicolumn{2}{|c|}{$\begin{array}{l}\text { Stade de la } \\
\text { menace } \\
\text { financière }\end{array}$} & $\begin{array}{l}\text { Stade de l'intro- } \\
\text { duction de nouv. } \\
\text { produits dans le } \\
\text { marché initial }\end{array}$ & $\begin{array}{l}\text { ro- Stade du } \\
\text { uv. désengage- } \\
\text { le ment ou de la } \\
\text { renégociation }\end{array}$ \\
\hline \multirow[t]{2}{*}{$\begin{array}{l}\text { M. Basire } \\
\text { (1976): }\end{array}$} & \multicolumn{2}{|c|}{$\begin{array}{l}\text { Stade 1a } \\
\text { primauté à la } \\
\text { fabrication }\end{array}$} & \multicolumn{3}{|c|}{$\begin{array}{l}\text { Stade } 1 b \\
\text { développement } \\
\text { des fonctions } \\
\text { commerciale et } \\
\text { administrative }\end{array}$} & \multicolumn{2}{|c|}{$\begin{array}{l}\text { Stade 1c } \\
\text { développement de } \\
\text { la planification }\end{array}$} & $\begin{array}{l}\text { Stade 1d } \\
\text { systématisation } \\
\text { des prévisions } \\
\text { et dével. fonct. } \\
\text { financier }\end{array}$ \\
\hline & \multicolumn{2}{|c|}{$\begin{array}{l}\text { Stade } 2 \\
\text { nomination d'un } \\
\text { directeur } \\
\text { commercial }\end{array}$} & \multicolumn{3}{|c|}{$\begin{array}{l}\text { Stade } 3 \\
\text { nomination d'un } \\
\text { directeur de } \\
\text { production }\end{array}$} & \multicolumn{2}{|c|}{$\begin{array}{l}\text { Stade } 4 \\
\text { nomination d'un } \\
\text { direct. fonction } \\
\text { prév. et financière }\end{array}$} & $\begin{array}{l}\text { Stade } 5 \\
\text { nomination d'un } \\
\text { directeur du } \\
\text { personnel }\end{array}$ \\
\hline $\begin{array}{l}\text { L. Hosmer } \\
\text { (1977): }\end{array}$ & \multicolumn{2}{|c|}{$\begin{array}{l}\text { Stade du fondateur } \\
\text { opérant }\end{array}$} & \multicolumn{2}{|c|}{$\begin{array}{l}\text { Début de } \\
\text { croissance }\end{array}$} & & \multicolumn{2}{|c|}{$\begin{array}{l}\text { Direction par } \\
\text { délégation }\end{array}$} & $\begin{array}{l}\text { Direction et } \\
\text { gestion à ni- } \\
\text { veaux multiples }\end{array}$ \\
\hline $\begin{array}{l}\text { J. Susbauer } \\
\text { (1979): }\end{array}$ & \multicolumn{2}{|c|}{$\begin{array}{l}\text { De survie (petit } \\
\text { marché, sous } \\
\text { capitalisée) }\end{array}$} & \multicolumn{3}{|c|}{$\begin{array}{l}\text { De croissance } \\
\text { (gestion profession- } \\
\text { nelle et financière }\end{array}$} & \multicolumn{2}{|c|}{$\begin{array}{l}\text { Sous-réalisée (grand } \\
\text { potentiel freiné) }\end{array}$} & $\begin{array}{l}\text { A grand succès } \\
\text { (grand potentiel } \\
\text { recherché) }\end{array}$ \\
\hline $\begin{array}{l}\text { N. Ibnabdeljalil } \\
\text { (1980): } \\
\text { faible croissance) }\end{array}$ & \multicolumn{3}{|c|}{$\begin{array}{l}\text { "Vivrières ou } \\
\text { rentières" (à } \\
\text { maîtrisée) }\end{array}$} & \multicolumn{3}{|c|}{$\begin{array}{l}\text { «Fragiles» (à } \\
\text { croissance mal } \\
\text { soutenue) }\end{array}$} & \multicolumn{2}{|c|}{$\begin{array}{l}\text { «Performantes» } \\
\text { (à croissance }\end{array}$} \\
\hline $\begin{array}{l}\text { N. Churchill } \\
\text { et V. Lewis } \\
(1983, T)\end{array}$ & $\begin{array}{l}\text { Existence } \\
\text { (prop. unique } \\
\text { centralisée, } \\
\text { marchés lim.) }\end{array}$ & \multicolumn{2}{|c|}{$\begin{array}{l}\text { Survie (début } \\
\text { décentral. } \\
\text { marchés plus } \\
\text { complexes) }\end{array}$} & $\begin{array}{l}\text { Succès } \\
\text { (profits } \\
\text { structure } \\
\text { fonctionr }\end{array}$ & inelle) & \multicolumn{2}{|c|}{$\begin{array}{l}\text { Envol (déléga- } \\
\text { tion, divisions, } \\
\text { marchés matures) }\end{array}$} & $\begin{array}{l}\text { Grande E. } \\
\text { (croissance, } \\
\text { diversifica- } \\
\text { tion, etc.) }\end{array}$ \\
\hline
\end{tabular}


Dans certains cas, on peut comprendre que les auteurs ne parlent que de l'évolution des firmes, d'artisanales à petites et de petites à moyennes. Nous avons dans ce cas Thain (1969) et Webster (1976). On peut utiliser aussi les typologies de Steinneetz (1969), Kroeger (1974), Basire (1976), Hosmer (1977) ou Susbauer (1979) pour y voir une évolution, soit à l'intérieur du concept de PME, soit à celui de petite à grande entreprise.

Malgré cette importante limite, l'idée de l'évolution de la structure, à mesure que l'entreprise grandit, est intéressante. Même si, comme nous l'avons dit, le point d'arrivée organisationnel peut différer considérablement d'une PME à l'autre, le schéma de M. Basire, avec sa subdivision en quatre parties du premier stade, est particulièrement utile pour évaluer l'évolution structurelle des PME. L'avantdernière typologie de ce groupe, celle de Ibnabdeljalil (1980), est montrée ici à titre d'exemple d'un grand nombre de typologies reliées aux aspects financiers selon les différents stades des entreprises.

\subsection{Typologies selon les secteurs, les types ou les opportunités de marché}

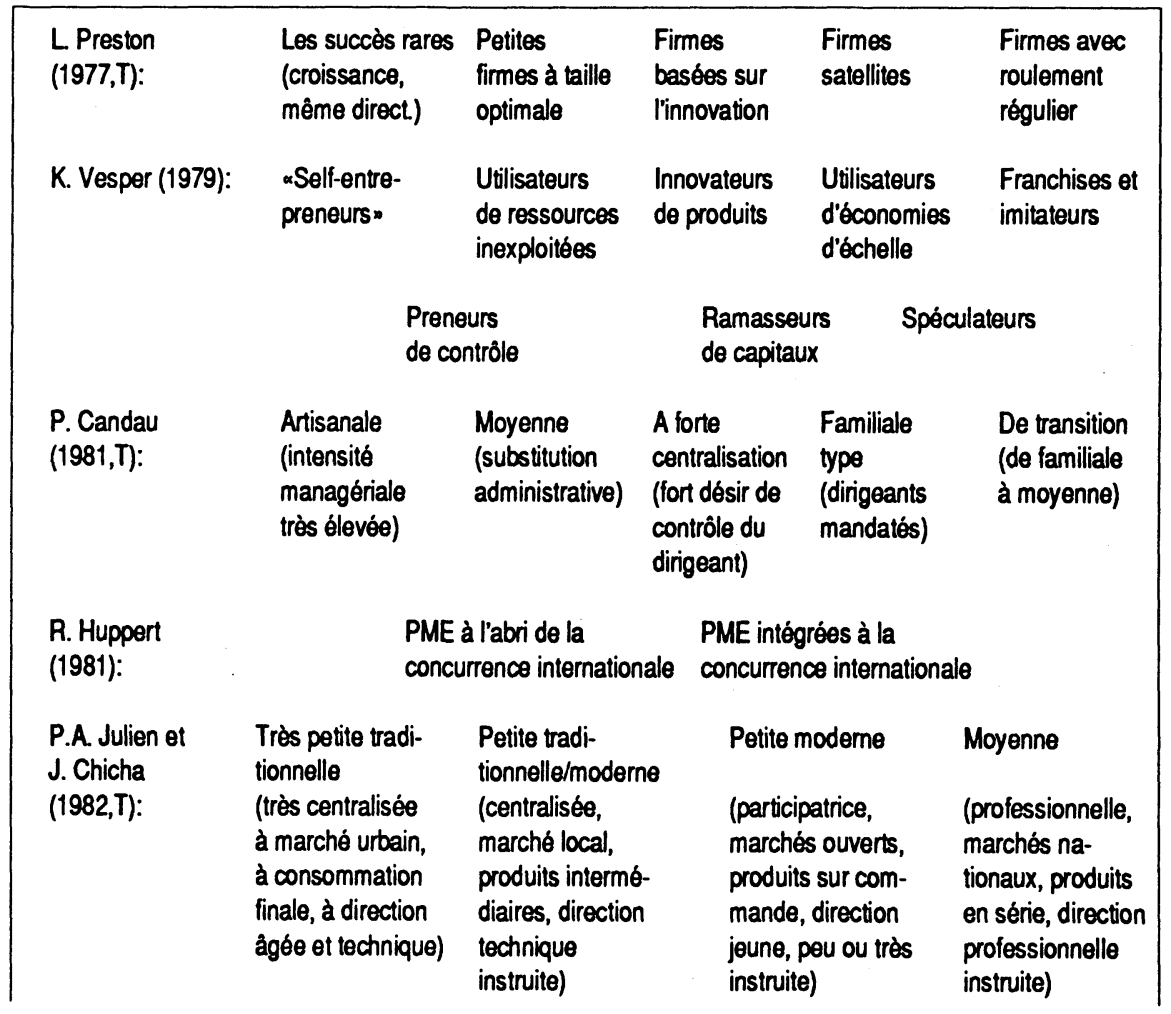




$\begin{array}{lllll}\text { C. Potier } & \text { De main-d'oeuvre } & \text { De production } & \text { De métiers } & \text { A technologie } \\ \text { (1986): } & \text { secteurs tradi- } & \text { spécifique } & \text { (sous-traitance de } & \text { de pointe } \\ & \text { tionnels (à façon, } & \text { (de savoir-faire, } & \text { capacité, de spé- } \\ & \text { roulement élevé...) } & \text { haut-de-gamme) } & \text { cialité) }\end{array}$

$\begin{array}{lllll}\text { A. Rizzoni } & \text { PME passi- } & \text { PME «tra- } & \text { PME dominées PME «imitative» } \\ \text { (1988): } & \text { ve (secteur } & \text { ditionnelle- } & \text { ou liées (à la } & \text { (flexibilité, } \\ & \text { mature, } & \text { (créneaux, } & \text { GE, tochnolo- } & \text { technologie +éle- } \\ & \text { marché local } & \text { ou mature } & \text { gie moyenne } & \text { vée, structure } \\ & \text { survie) } & \text { m. régional } & \text { crois. lente, } & \text { +complexe) } \\ & & \text { centralisée } & \text { centralisée) } & \\ & & \text { stabilité) }\end{array}$

PME à base PME de pointe technolo- (entr. technogique logique,+pro(entre. fessionnel, schumpete- innovation rien, inno- radicale vation non radicale, croissance)

Il existe beaucoup d'autres typologies, telles les typologies concernant la situation financière (Huret, 1974; Grégoire et Harrière, 1976; Mader, 1979; Robitaille et Marchesnay, 1988) ou discutant de l'intensité capitalistique (Eymard-Duvernay et Delattre, 1983). Nous n'avons pas abordé, non plus, les typologies particulières sur l'artisanat (Zarka, 1979; Jaeger, 1982; Arena et alii, 1985) ou sur les petites firmes «alternatives» (Joyal, 1988). Notre intention n'était pas de faire une recension aussi exhaustive que possible; les typologies retenues ici suffisent pour démontrer toute la complexité du problème et ainsi la grande hétérogénéité des PME.

\section{$4 \quad$ Vers une typologie complexe globale}

La plupart des typologies retenues touchent divers éléments qu'on retrouve dans les PME (ou du moins dans les petites entreprises ayant plus de cinq employés) et surtout dans les PME manufacturières ou PMI. Il existe encore peu de typologies qualitatives s'appliquant aux secteurs commercial et des services.

Pour résumer ces diverses typologies, on peut disposer leurs différents éléments sur plusieurs «continuum», allant de moins à plus, soit :

Précisons toutefois que cette optique est encore trop simple. S'il y a des "continua», ceux-ci ne sont pas nécessairement linéaires. On peut voir apparaître des «sauts» à mesure que l'on passe d'une taille à l'autre. Par exemple, l'organisation ne se complexifiera pas graduellement ou de la même façon selon le type de fonction. Ainsi, le dirigeant de la très petite entreprise manufacturière qui voit sa production augmenter, engage d'abord un contremaître ou un responsable de la production, puis recourt à un comptable; ce n'est que plus tard qu'il met en place un ou deux niveaux de gestion dans la fonction marketing, tout en conservant le contrôle des achats et du personnel; etc. Dans les faits, ce continuum organisationnel se présentera plutôt ainsi : 
Fig. 1 Typologle sur «continuum»

19 La dimension brute

nombre d'employés, actifs, chiffre d'affaires ou de ventes

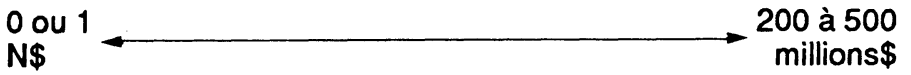

$2^{9} \quad$ Le secteur

de traditionnel ou mature ou à produits pour les

consommateurs

à secteurs modernes ou à produits

$\cdots$ secondaires, ou de pointe (selon les définitions)

30 Le marché

local international protégé ouvert

$4^{2}$ Contrôle et organisation

centralisée décentralisée à un ou plusieurs niveaux

indépendance forte liée (satellite)

$5^{2} \quad$ Stratégie, objectifs

intuitive formalisée

de survie, à faible risque de croissance, à haut risque

69 Technologie et innovation traditionnelle, mature innovation faible incrémentale

Fig. 2 Evolution des niveaux de gestion

Centralisée Décentralisée

1 ou 2 niveaux dans telle fonction

$x$ niveaux dans telle fonction plusieurs niveaux dans toutes les fonctions 
Suivant l'optique envisagée ou la discipline privilégiée, les typologies mettront plus d'accent sur un aspect en particulier, sur plusieurs ou sur quelques-uns seulement.

On peut penser que les petites et les très petites entreprises se retrouveront plutôt sur les segments vers la gauche, avec des différences sur certains «continua» selon les secteurs, les marchés, la direction...; alors que les moyennes entreprises apparaîtront plutôt vers la droite (plus évident encore dans le cas des grandes et des très grandes entreprises), avec, une fois de plus, diverses différences dues justement à la grande hétérogénéité des PME.

Ces divers «continua» réfêrent donc à diverses caractéristiques de la PME. Se basant, à un titre ou à un autre, sur diverses études que nous avons réalisées et en tenant compte d'autres auteurs que nous venons d'analyser, nous avons retenu les six caractéristiques suivantes pour mieux cerner le concept de PME, soit :

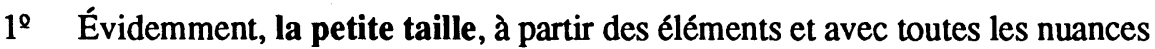
dont nous avons parlé plus haut.

$2^{\circ} \quad$ La centralisation de la gestion. On peut même parler de «personnalisation» de la gestion en la personne du propriétaire- dirigeant dans le cas de toutes petites entreprises. Mais on peut aussi retrouver une forte centralisation dans des moyennes entreprises des secteurs traditionnels, comme l'ont montré Candau ou Rizzoni;

$3^{2} \quad$ Une faible spécialisation, tant au niveau de la direction (celle-ci assumant des tâches tant de direction que d'exécution, surtout lorqu'elle est petite) que des employés et des équipements. La spécialisation vient avec une plus grande taille, le passage de production variée, sur commande ou de très petites séries à une production en série et répétitive. C'est ainsi qu'à mesure que la firme grossit, elle doit mettre sur pied plusieurs niveaux organisationnels, d'abord, comme nous l'avons dit, dans les fonctions comptable et de production, ensuite, dans la fonction marketing, puis plus tard, dans celles des achats, de la R \& D et enfin du personnel.

$4^{9} \quad$ Une stratégie intuitive ou peu formalisée. Alors que dans les grandes entreprises on doit préparer des «plans» relativement précis des actions à venir pour que toute la direction puisse s'y référer, dans les petites entreprises le propriétaire-dirigeant est suffisamment proche de ses employés clés pour leur expliquer, au besoin, tout changement de direction.

$5^{\varrho} \quad$ Un système d'information interne simple ou peu organisé. Les petites organisations fonctionnent par dialogue ou par perception directe. Les grandes doivent mettre sur pied tout un mécanisme formel (et écrit) permettant la circulation d'informations tout en minimisant le «bruit» et en favorisant le contrôle. Les très grandes entreprises vont même jusqu'à produire leur propre «journal» pour diffuser l'information générale et empêcher que les rumeurs nuisent à l'efficacité de l'entreprise. 
$6^{9} \quad$ Enfin un système d'information externe simple. Dans les entreprises artisanales, le propriétaire-dirigeant peut discuter directement avec ses clients tant pour connaître leurs besoins et leurs goûts que pour expliquer différents aspects du ou des produits. La grande organisation doit commander des études de marché coûteuses et complexes qui, souvent d'ailleurs, finissent par influencer la stratégie alors que la réalité a déjà changé. Il arrive cependant que certaines petites entreprises font face à des oligopsones (par exemple les groupements d'achat qui servent de tampon entre les PMI et les consommateurs) qui les coupent de leur marché véritable et ainsi de cette information directe.

Encore une fois, ces caractéristiques peuvent être vues sur un continuum, du moins au plus.

\section{Conclusion}

A la lumière de cette analyse, on peut penser qu'on dispose d'un bon bagage de typologies pour répondre à la plupart des besoins. Mais il reste encore bien des points obscurs.

Ces typologies n'ont pas encore été suffisamment bien testées selon divers marchés ou diverses conjonctures. De plus, comme on l'a dit, on manque encore de typologies opérationnelles pour les PME commerciales et de services. Aussi, les changements en cours dans le monde des PME, telle l'intrusion croissante du service dans le produit, entraînent de nouveaux types de PME encore trop peu étudiés.

Bref, il reste encore un champ très vaste de recherches à réaliser.

\section{Bibliographie}

Barry, S., (1978), «Organization design in the smaller enterprise», dans D.T. Bryault et R.J. Niehaus, Manpower, Planning and Organization, New York, Plenum Press.

Basire, M., (1976), «La théorie des cinq niveaux», Direction et gestion, $\mathrm{n}^{\mathrm{os}} 2 / 3 / 4$.

Bolton, J.E., (1971), Report of the Committee on Inquiry on Small Firms, Editeur officiel, Londres.

Brown, S.H. et Philips, B.D., (1989), «Comparisons between Small Business Data Base (USEEM) and Bureau of Labor Statistics (BSL) Employment Data : 1978-1986», Small Business Economics, vol. 1, p. 273-284.

Carland J., Hoy, F., Boulton, W. et Carland, J.A., (1984), «Differentiating entrepreneurs form small business owners : a conceptualization», Academy of Management Review, vol.9, p. 354-359.

Candau, P., (1981), «Pour une taxonomie de l'hypofirme», Revue d'économie industrielle, $\mathrm{n}^{\circ}$ 16. 
Cooper A. et Dunkelberg, C., (1982), «Entrepreneurial typologies : an empirical study», dans K. Vesper (éd.), Frontiers of Entrepreneurial Research, Université de Washington.

Churchill, N. et Lewiss, V., (1983), «Les cinq stades de l'évolution d'une P.M.E.», Harvardl'Expansion, automne, p. 51-63.

Cooper, A.C., (1979), «Strategic management : new venture and small business», dans Schendell et Hofer (éd.) Strategic Management : a New View of Business Policy and Planning, Boston.

Deeks, J., (1973), «The small firm. Asset or liability?», Journal of Management Studies, vol. $10, n^{\circ} 1$, p. $25-47$.

Filey, A. et Aldag, R., (1978), «Characteristics and measurement of an organizational typology», Academy of Management Journal, décembre.

Gélinier, O. et Gaultier, A., (1974), L'avenir des entreprises personnelles et familiales, Paris, Éditions Hommes et Techniques.

Greiner, L., (1972), «Evolution and revolution of organizations growth», Harvard Business Review, vol. 50, $\mathrm{n}^{\circ} 4$, p. 37-46.

Hosmer, L., Cooper, A.et Vesper, K., (1977), The Entrepreneurial Function, Englewood Cliffs, Prentice-Hall.

Houssiaux, J., (1957), «Le concept de quasi-intégration et le rôle des sous-traitants dans l'industrie», Revue Économique, mars.

Hupper, R., (1981), «Stratégies de développement des PMI françaises», Revue d'économie industrielle, $\mathrm{n}^{\circ} 17$.

Ibnabdeljalil, N., (1980), «Contribution à une analyse financière et typologique des PME industrielles», Thèse d'État, Université de Rennes, cité par J. Bernard et J.L. Ravix, «Diversité et spécificité des unités productives : les clivages par taille et les typologies d'entreprises", dans R. Arena, et alii. Traité d'économie industrielle, Paris, Economica, 1988.

Julien, P.A. et Chicha, J., (1982), «Vers une typologie multicritères des PMEmanufacturières», Cahiers de recherche du GREPME $n^{\circ}$ 82-06.

Kroeger, C., (1974), «Managerial development of the small firm», California Management Review, vol. $17, \mathrm{n}^{\circ} 1, \mathrm{p} .41-46$.

Lorrain, J. et Dusseault, L., (1988), «Les entrepreneurs artisans et opportunistes : une comparaison de leurs comportements de gestion», Revue Internationale P.M.E., vol. $1, \mathrm{n}^{\circ}$ 2, p. 157-177.

Liles, P., (1974), New Business Ventures and the Entrepreneur, Homewood, R.D. Irwin.

Mader, F., (1979), «Un échantillon d'entreprises en difficulté», Journée de la centrale de bilan, cité par Bernard et Ravik, op. cit..

Miller, D. et Friesen, P.H., (1982), «Innovation in conservative and entrepreneurial firms : two models of strategic momentum», Strategic Management Journal, vol. 3, $\mathrm{n}^{\circ} 1, \mathrm{p} .10-25$.

Mueler, D.C., (1972), «A life cycle theory of the firms», Journal of Industrial Economic, juin.

Nguyen, T.H. et Belhumeur, A., (1985), «A propos de l'interchangeabilité des mesures de taille d'entreprise», Revue d'économie industrielle, $\mathrm{n}^{\circ} 33$.

Pensors, E., (1952), «Biological analogies in the theory of the firm», American Economic Review, $\mathrm{n}^{\circ} 42$.

Paulson, S., (1980), «Organizational size, technology structure : replication of a study of social services agencies among small retail firms», Academy of Management Journal, juin.

Preston, L., (1977), «The world of small business : a suggested typology», American Journal of Small Business, avril. 
Quélennec, M., (1986), «Les statistiques d'entreprises : réalités observées et méthodes d'observations», Collection de l'INSEE, série no 101.

Rizzoni, A., (1988), «Innovazione tecnologica et piccola impresa : un approcio tipologico», Piccola Impresa, $\mathrm{n}^{\circ} 1, \mathrm{p} .67-89$.

Smith, N. et Miner, B., (1983), «Type of entrepreneur, type of firm and managerial motivation : implication for organizational life cycle theory», Strategic Management Journal, vol. 4, p. 325-340.

Stanworth, M. et Curran, J., (1976), «Growth and the small firm : and alternative view», Journal of Management Studies, mai.

Steinneetz, L., (1969), «Critical stages of small business», Business Horizons, février.

Storey, D.J., (éd.) (1983), The Small Firm : an International Survey, New York, St. Martin Press.

Susbauer, J., (1979), «Strategic management and organization types : commentary», dans Schendell et Hofer, op. cit.

Thain, D., (1969), «Stages of corporate development», Business Quarterly, hiver.

Vargas, G., (1984), «Les crises de croissance de la PMI-PME», Revue française de gestion, janvier-février.

Vesper, K., (1979), «Strategic management and organization types : commentary», dans Schendell et Hofer, op. cit..

Webster, F., (1976), «A model for new venture initiation», Academy of Management Review, vol. $1, \mathrm{n}^{\circ} 1$. 